\title{
Representations of molecular force fields. IV. Vibrational stress, isotope effects, and structure of ethane
}

\author{
L. S. Bartell and Susan Fitzwater
}

Department of Chemistry, The University of Michigan, Ann Arbor, Michigan 48109

(Received 11 March 1977)

\begin{abstract}
The transformation of molecular force fields from natural curvilinear coordinates $\tilde{\mathbf{S}}$ to rectilinear coordinates $\mathbf{S}$ (yielding simpler equations of motion) is discussed. Rectilinear cubic constants include mass dependent terms arising from the nonlinear coordinate transformation as well as contributions from the cubic constants for the $\tilde{\mathbf{S}}$ displacements. These contributors to anharmonicity lead to intramolecular "vibrational stresses" resolvable into components $\mathfrak{F}^{C}, F^{r}$, and $\mathscr{F}^{F}$ which induce rectilinear molecular strain components $\langle S\rangle_{C},\langle S\rangle_{T}$, and $\langle S\rangle_{F}$, each of which has its own physical interpretation. More significant than the rectilinear quantities are the curvilinear stresses and strains which, it is shown, are readily expressed in terms of the above components. Each of the foregoing quantities is easily decomposed into contributions from the various types of vibration. Magnitudes are illustrated by computations of cubic force constants and selected stress and strain components in $\mathrm{C}_{2} \mathrm{H}_{6}$ and $\mathrm{C}_{2} \mathrm{D}_{6}$.
\end{abstract}

\section{INTRODUCTION}

Because atomic positions always have a comparatively large natural indeterminacy $\left(\sim 10^{-1} \AA\right)$, the value as signed to a given internuclear distance depends critically upon the way the vibrational average is defined. Two of the most important averages are $r_{g}$ and $r_{z}$, the average internuclear distance and the distance between average internuclear positions, respectively. These averages, expressed by Morino, Kuchitsu, and coworkers ${ }^{1-3}$ as

$$
\text { and } \begin{aligned}
r_{g} & =r_{e}+\langle\Delta z\rangle+\left(\left\langle\Delta x^{2}\right\rangle+\left\langle\Delta y^{2}\right\rangle\right) / 2 r_{e}+\cdots \\
r_{z} & =r_{e}+\langle\Delta z\rangle
\end{aligned}
$$

are sensitive to magnitudes of both harmonic and anharmonic components of the molecular force field. Our growing experimental and theoretical knowledge of anharmonic potential constants makes it increasingly worthwhile to pay heed to vibrational effects. One of the most significant recent advances in anharmonic molecular vibration theory has been the introduction of the I tensor formalism by Hoy, Mills, and Strey. ${ }^{4}$ Another approach, only roughly worked out in its initial application, is a method focusing on intramolecular vibrational stresses. ${ }^{5}$ This method is attractive in the way it can be reduced to physically interpretable components of anharmonic atomic displacements and isotope effects on mean internuclear distances. The object of the present paper is to combine the instructiveness of the vibrational stress picture with the rigor of the $L$ tensor formalism, and to illustrate the approach with ethane and perdeuteroethane as examples. For simplicity we shall carry out the treatment only through the effects of cubic force constants. Because the present treatment corresponds to a transformation of the Hoy, Mills, and Strey results back to internal coordinates, it can be extended to arbitrary order in the framework of the $L$ tensor formulation. By the same token, it yields no information not derivable, in principle, from the $L$ tensor approach.

\section{THEORY}

It is essential to distinguish carefully between the rectilinear internal coordinates $R$ constructed from
Cartesian displacements and the natural curvilinear internal coordinates designated by Mills et al. ${ }^{4}$ by a Russian "ya" but in the present work (where ya cannot be typeset) by $\tilde{R}$. In the following a tilde will be used to identify symbols pertaining to curvilinear coordinates. For the purposes of the present paper we shall carry out calculations in terms of the symmetry coordinates

$$
S_{i}=\sum_{j} U_{i j} R_{j}
$$

and

$$
\tilde{S}_{i}=\sum_{j} U_{i j} \tilde{R}_{j},
$$

which, of course, can be taken simply as the internal coordinates, if desired. The most fundamental expression for the force field, as emphasized by Mills et al. , ${ }^{4}$ is in terms of the $\tilde{R}$ coordinates, or

$V=1 / 2 \sum_{i} \sum_{j} f_{i j} \tilde{S}_{i} \tilde{S}_{j}+1 / 6 \sum_{i} \sum_{j} \sum_{k} \tilde{f}_{i j k} \tilde{S}_{i} \tilde{S}_{j} \tilde{S}_{k}+\cdots$

rather than

$$
V=1 / 2 \sum_{i} \sum_{j} f_{i j} S_{i} S_{j}+1 / 6 \sum_{i} \sum_{j} \sum_{k} f_{i j k} S_{i} s_{j} S_{k}+\cdots
$$

because the $f_{i j k}$, unlike the $\tilde{f}_{i j k}$, depend upon the atomic masses involved (as a result of the Ekart conditions imposed in the definitions of the R). A particularly convenient way of deducing mean displacements in internuclear distances is to apply Ehrenfest's theorem ${ }^{6}$

$$
m d^{2}\left\langle x_{k}\right\rangle / d t^{2}=-\left\langle\partial V / \partial x_{k}\right\rangle, \text { etc. , }
$$

according to which, for stationary states or equilibrium distributions among stationary states, the acceleration of Cartesian coordinates is zero, giving the set of equations $^{7}$

$$
\left\langle\partial V / \partial S_{i}\right\rangle=0 \text {. }
$$

By carrying out the indicated differentiations upon Eq. (5) the resulting relations

$$
\sum_{j} f_{i j}\left\langle S_{j}\right\rangle+1 / 2 \sum_{j} \sum_{k} f_{i j k}\left\langle S_{j} S_{k}\right\rangle+\cdots=0
$$


can be solved for the displacements $\left\langle S_{j}\right\rangle$ in terms of the relatively simple mean square displacements $\left\langle S_{j} S_{k}\right\rangle$. In matrix notation Eq. (8) is expressible as

$$
\mathbf{f}\langle\mathbf{S}\rangle=\mathfrak{F}
$$

according to which.

$$
\left\langle S_{k}\right\rangle=\sum_{l}\left(f^{-1}\right)_{k l} F_{i},
$$

where the elements of the column matrix $\langle\mathbf{S}\rangle$ are the mean displacements of the totally symmetric symmetry coordinates (reckoned relative to the equilibrium $\left[\boldsymbol{r}_{e}\right]$ structure) and the column matrix $\mathcal{F}$ has elements

$$
\mathcal{F}_{i}=-1 / 2 \sum_{i} \sum_{j} f_{i j l}\left\langle S_{i} S_{j}\right\rangle+\cdots \cdot
$$

By virtue of the resemblance of Eq. (9) to Hooke's law, the elements $\mathcal{F}_{1}$ can be interpreted as intramolecular vibrational stresses arising from the curved trajectories of atoms vibrating in the skewed (anharmonic) wells of the potential function. Although the original paper ${ }^{7}$ pointed out that the application of Eq. (7) is appropriate for rectilinear but not curvilinear coordinates, the initial treatment ${ }^{5}$ of secondary isotope effects disregarded this limitation and, accordingly, obtained equivocal results. In the following we show the relationship between the vibrational stresses for the rectilinear coordinates and those for the curvilinear coordinates.

Crucial to the problem is the nonlinearity in the transformation between the $R$ and the $\vec{R}$ coordinates. Even if the cubic constants $\tilde{f}_{i j k}$ for the $\tilde{\mathbf{R}}$-based coordinates [Eq. (4)] were zero, nonlinearities would propagate quadratic potential constants into the rectilinear cubic constants $f_{i j k}$ as follows: From the transformation ${ }^{4}$

$$
\tilde{S}_{r}=S_{r}+1 / 2 \sum_{i} \sum_{j} T_{r}^{i j} S_{i} S_{j}+\cdots
$$

expressing the aforementioned nonlinearity in the coordinate transformation it follows that the rectilinear cubic constants are

$$
\begin{aligned}
f_{i j l} & =\tilde{f}_{i j l}+\sum_{r}\left(f_{r i} T_{r}^{l j}+f_{r j} T_{r}^{i l}\right)+\sum_{r} f_{r l} T_{r}^{i j} \\
& \equiv \tilde{f}_{i j l}+f_{i j l}^{T}+f_{i j l}^{F} .
\end{aligned}
$$

As Eq. (13) stands there is no significance in the subdivision into " $T$ " and " $F$ " components since $i, j$, and $l$ all enter Eq. (13) on the same footing. In deriving the vibrational stress component acting on the $l$ th coordinate, however, a distinction between $l$ and $i, j$ is made which makes it worthwhile to decompose $f_{i j l}$ as shown. This decomposition is profitably continued through stress and strain components as follows: Vibrational stress components $F_{l}$ of Eq. (11) can be written, in view of Eq. (13), as

$$
\mathfrak{F}_{l}=\mathfrak{F}_{l}^{C}+\mathcal{F}_{l}^{T}+\mathfrak{\Im}_{l}^{F},
$$

where $F_{i}$ is derived from the cubic constants $\tilde{f}_{i j l}$ of the curvilinear coordinates, and the other components originate from the nonlinear transformation. The mean rectilinear coordinates can be similarly broken down, by inserting Eq. (14) into (10), to yield

$$
\langle\mathbf{S}\rangle=\langle\mathbf{S}\rangle_{C}+\langle\mathbf{S}\rangle_{T}+\langle\mathbf{S}\rangle_{\boldsymbol{F}}
$$

It can be readily verified that the last component of $\langle\mathbf{S}\rangle$ reduces to

$$
\left\langle S_{k}\right\rangle_{F}=-1 / 2 \sum_{i} \sum_{j} T_{k}^{i j}\left\langle S_{i} S_{j}\right\rangle .
$$

This result, by virtue of the definition of Eq. (12) (space-averaged over both sides), demonstrates the special significance of $\langle\mathbf{S}\rangle_{F}$, namely,

$$
\langle\mathbf{S}\rangle_{F}=-(\langle\overline{\mathbf{S}}\rangle-\langle\mathbf{S}\rangle)
$$

It is apparent that the difference $\left(\left\langle\tilde{S}_{k}\right\rangle-\left\langle S_{k}\right\rangle\right)$ between mean curvilinear and rectilinear coordinates is the foreshortening correction required to express the effect of perpendicular amplitudes of vibration. This correction reduces, if $\vec{S}_{k}$ represents a bond stretch (e.g., $\tilde{S}_{3}=\Delta r_{\mathrm{CC}}$ in ethane), to the familiar

$$
r_{s}-r_{z}=\left(\left\langle\Delta x^{2}\right\rangle+\left\langle\Delta y^{2}\right\rangle\right) / 2 r_{e}
$$

of Eq. (1) and the equivalent

$$
r_{g}-r_{z}=1 / 2 \sum_{s} L_{k}^{s s}\left\langle Q_{s}^{2}\right\rangle
$$

of Mills. ${ }^{8}$ Since this result is obtained even if a bond is completely rigid, the utility of separating the foreshortening correction $\langle S\rangle_{F}$ from the other "transformation" correction $\langle\mathbf{S}\rangle_{r}$ and from the cubic correction $\langle\mathbf{S}\rangle_{C}$ is obvious. Equation (17) is perfectly general and applies as well to bond angles and dihedral angles. Higher order corrections arising from terms dropped in the truncations specified in Eqs. (8) and (11) are discussed, for Morse oscillators, in Ref. 7. Also examined in the same reference is the difference between using zeroth-order mean square amplitudes and exact mean square amplitudes.

In view of Eqs. (14)-(17) it is possible to write a vibrational stress matrix $\overline{\mathcal{F}}$ applying directly to the curvilinear coordinates, or

$$
\mathbf{f}\langle\tilde{\mathbf{S}}\rangle=\tilde{\mathfrak{F}}
$$

where stress elements $\tilde{F}_{l}$ are comprised of the first two rhs terms of Eq. (14), namely,

$$
\overline{\mathfrak{F}}_{l}=\mathfrak{F}_{l}^{C}+\mathfrak{F}_{l}^{T} \text {. }
$$

Thus, the mean curvilinear displacements can be written as

$$
\begin{aligned}
\left\langle\tilde{S}_{k}\right\rangle & =\sum_{l}\left(f^{-1}\right)_{k l} \bar{F}_{l} \\
& =\left\langle S_{k}\right\rangle_{C}+\left\langle S_{k}\right\rangle_{T},
\end{aligned}
$$

where the components $\left\langle S_{k}\right\rangle_{C}$ and $\left\langle S_{h}\right\rangle_{T}$ are the same as those in Eq. (15). Since $\left\langle S_{k}\right\rangle_{c}$ is derived from the cubic constants $f_{i j l}$ of the curvilinear coordinates in the same manner as the rectilinear mean $\left\langle S_{k}\right\rangle$ is derived from the cubic constants $f_{i j l}$ of the rectilinear coordinates, the fact that $\left\langle S_{h}\right\rangle_{T}$ is nonvanishing is a manifestation that the simple Newtonian average in Eq. (6) holds only if $x_{k}$ is rectilinear. The physical meaning of the transformation correction $\left\langle S_{k}\right\rangle_{T}$ is in many cases straightforward. For example, if $S_{k}$ represents the stretch of a bond whose terminal atoms are oscillating over nearly circular 
TABLE I. Cubic force constants for ethane; $\tilde{f}$ and $f$ are for curvilinear and rectilinear coordinates, respectively; $\mathrm{H}$ and $\mathrm{D}$ represent $\mathrm{C}_{2} \mathrm{H}_{6}$ and $\mathrm{C}_{2} \mathrm{D}_{6}$, respectively.

\begin{tabular}{lcrrrrrr}
\hline$i j k$ & $\bar{f}_{i j k}{ }^{\mathrm{a}}$ & $(f-\tilde{f})_{i j k}^{\mathrm{H}}$ & $\left(f^{\mathrm{H}}-f^{\mathrm{D}}\right)_{i j k}$ & $i j k$ & \multicolumn{1}{c}{$f_{i j k}$} & $\left(f-f_{i j k}^{\mathrm{H}}\left(f^{\mathrm{H}}-f^{\mathrm{D}}\right)_{i j k}\right.$ \\
\hline 111 & $-12.52(6)$ & 0.00 & 0.00 & 882 & $-0.35(2)$ & 0.25 & 0.00 \\
221 & 0.04 & 0.52 & 0.00 & 992 & $0.18(4)$ & -0.07 & 0.01 \\
231 & $\cdots$ & 0.18 & 0.00 & $10,10,2$ & {$[-0.07]$} & -0.20 & 0.07 \\
331 & $-2.96(30)$ & 0.00 & 0.00 & $11,11,2$ & $-0.35(2)$ & 0.22 & -0.02 \\
551 & {$[-12.52]$} & 0.00 & 0.00 & $12,12,2$ & $0.26(4)$ & -0.09 & -0.04 \\
661 & {$[0.04]$} & 0.69 & 0.02 & 113 & $0.29(0)$ & 0.00 & 0.00 \\
771 & {$[-12.52]$} & 0.00 & 0.00 & 223 & $-0.52(0)$ & 0.15 & 0.00 \\
881 & $\cdots$ & 0.58 & -0.01 & 333 & $-23.45(1)$ & 0.00 & 0.00 \\
991 & $\ldots$ & 2.12 & 0.09 & 443 & $-0.33(1)$ & -0.13 & $\cdots$ \\
$10,10,1$ & {$[-12.52]$} & 0.03 & -0.01 & 553 & $0.29(6)$ & 0.00 & 0.00 \\
$11,11,1$ & $\cdots$ & 0.47 & -0.04 & 663 & $-0.34(1)$ & 0.14 & -0.02 \\
$12,12,1$ & $\ldots$ & 1.02 & 0.26 & 773 & $0.26(6)$ & 0.00 & 0.00 \\
112 & -0.07 & -0.04 & 0.00 & 883 & $0.03(6)$ & -0.01 & -0.02 \\
222 & $0.49(1)$ & -0.55 & 0.00 & 993 & $-0.93(1)$ & 0.02 & -0.03 \\
332 & $0.90(4)$ & 0.00 & 0.00 & $10,10,3$ & $0.32(6)$ & -0.02 & 0.00 \\
552 & {$[-0.07]$} & -0.04 & 0.00 & $11,11,3$ & $0.09(6)$ & -0.03 & -0.04 \\
662 & $0.56(10)$ & -0.55 & 0.00 & $12,12,3$ & $-0.91(1)$ & -0.11 & -0.01 \\
772 & {$[-0.07]$} & 0.03 & 0.01 & & & &
\end{tabular}

aAll constants except for $\tilde{f}_{112}, \tilde{f}_{221}$, and those in square brackets are from the ab initio calculations of Ref. 9. Constants $f_{112}$ and $f_{221}$ were calculated for this study by similar methods. Bracketed constants are assumed to be approximately equal to the corresponding values for related modes. Energy in mdyn $\AA$ ( $=10^{-18} \mathrm{~J}$ ), displacements in $\AA$ for stretches, rad for bends. Uncertainties are indicated in parentheses. Because of minor inconsistencies mentioned in Sec. III, curvilinear to rectilinear corrections in $f_{i j k}$ are uncertain by perhaps 0.02 units.

arcs, $\left\langle S_{k}\right\rangle_{T}$ is the stretch induced by the mean centrifugal reactions in the curved trajectories. ${ }^{7}$

From the foregoing it is clear that the vibrational stress components $\widetilde{F}_{7}$ and $\tilde{F}_{l}$ can be determined for various coordinates $S_{l}$ or $\tilde{S}_{l}$ in a straightforward way. It should be emphasized that the $\tilde{S}_{l}$ correspond much more directly to physical observations than do the $S_{l}$ because the former correspond always to geometrical measurements in a molecule whereas the latter involve the Eckart conditions. In the absence of displacements of the other coordinates the mean displacement of a given coordinate, for example $\tilde{S}_{l}$, is given simply by $\bar{F}_{l} / f_{l l}$ according to Eq. (18). Displacements of other coordinates $\tilde{S}_{m}$ propagate effects into the $\tilde{S}_{l}$ coordinates through the couplings implicit in the $f_{l m}$ as shown in Eq. (20). A resolution of the stress components into contributions originating from the various amplitudes of vibration is frequently illuminating, as illustrated in Ref. 5 .

Hoy, Mills, and Strey ${ }^{4}$ have outlined several ways to determine the transformation tensor elements $T_{r}^{i j}$ that are needed in the foregoing treatment. Their relation

$$
T_{r}^{i j}=\sum_{m} \sum_{n} L_{r}^{m n}\left(L^{-1}\right)_{m}^{i}\left(L^{-1}\right)_{n}^{j}
$$

is perhaps as convenient as any, although the tensor can be derived from considerations of mass and geometry without performing a normal coordinate analysis.

\section{APPLICATION TO ETHANE}

In order to illustrate the magnitudes involved both in the effects of the nonlinearities of the transformations and in the dependence of the cubic constants $f_{i j k}$ upon atomic masses we present selected results for the cases of $\mathrm{C}_{2} \mathrm{H}_{6}$ and $\mathrm{C}_{2} \mathrm{D}_{6}$. For this system most of the required cubic force constants $\tilde{f}_{i j k}$ are available from an $a b$ initio calculation. ${ }^{9}$ These are displayed in Table I along with the corrections $(f-\tilde{f})_{i j k}$, and $\left(f^{\mathrm{H}}-f^{\mathrm{D}}\right)_{i j k}$ derived from Eq. (13). For practical reasons the quadratic 1 matrix and $L$ matrix were taken from Duncan's experimental study ${ }^{10}$ rather than from the $a b$ initio calculation. For this reason, because of the incompleteness of the set of $\tilde{f}_{i j k}$, and because a considerable amount of the computation was carried out by hand rather than by an efficient computer code (so that minor inconsistencies were tolerated in reference geometries in different phases of calculations) tabulated corrections are illustrative rather than final. These minor flaws should have no significant effect on the magnitudes.

From Table I it can be seen that the distinction between the curvilinear and rectilinear cubic constants is small for the pure stretch constants although it is significantly nonzero for the 10,10,1 and 10,10,3 cases. Here, the $E_{g}$ stretch rocks the molecule and contributes to the bond foreshortening associated with the $(f-\tilde{f})$ correction. The largest curvilinear to rectilinear cubic corrections and the largest mass depen-

TABLE II. Ground state vibrational stress components ${ }^{\mathrm{a}}$ and mean displacements of rectilinear $A_{1 s}$ symmetry coordinates in ethane and deuteroethane.

\begin{tabular}{lllll}
\hline$k$ & $\mathcal{F}_{k}^{\mathrm{H}}$ & $\mathcal{T}_{k}^{\mathrm{D}}$ & $\left\langle S_{k}\right\rangle^{\mathrm{H}}$ & $\left\langle S_{k}\right\rangle^{\mathcal{D}}$ \\
\hline 1 & 0.1050 & 0.0699 & 0.0212 & 0.0141 \\
2 & 0.0077 & 0.0055 & 0.0236 & 0.0183 \\
3 & 0.0654 & 0.0533 & 0.0173 & 0.0140 \\
\hline \hline
\end{tabular}

$\mathrm{a}_{\Im_{k}}$ in mdyn $\AA\left(=10^{-18} \mathrm{~J}\right)$ per displacement $S_{k}(\AA$ for $k=1,3, \mathrm{rad}$ for $k=2$ ). Nonlinear transformation corrections for torsion have been neglected in the $\xi_{k}$. 
TABLE III. Ground state contributions from various vibrations to selected $\mathrm{C}-\mathrm{C}$ stresses and strains in $\mathrm{C}_{2} \mathrm{H}_{6}$ illustrating rectilinear to curvilinear corrections.

\begin{tabular}{|c|c|c|c|c|}
\hline Source & $\mathfrak{F}_{3}^{C}$ & $v_{3}^{T}$ & $\Im_{3}^{F}$ & $\left\langle\left(\tilde{S}_{3}-S_{3}\right)\right\rangle$ \\
\hline $\mathrm{HCC}$ bend & 41.79 & 5.27 & -7.37 & 0.48 \\
\hline $\mathrm{HCH}$ bend $\mathrm{d}^{\mathrm{a}}$ & -2.05 & 0.96 & 0.05 & 0.08 \\
\hline $\mathrm{CH}$ stretch & -5.45 & -0.09 & 0.21 & 0.02 \\
\hline CC stretch & 30.64 & 0.00 & 0.00 & 0.00 \\
\hline Torsion & 5.67 & 0.00 & 2.31 & 0.00 \\
\hline Cross terms & $-3.30^{b}$ & $-0.03^{b}$ & 0.21 & -0.01 \\
\hline Total & $\begin{array}{l}67.30 \\
10^{-3} \mathrm{mdyn}\end{array}$ & $\begin{array}{c}6.11 \\
10^{-3} \\
\mathrm{mdyn}\end{array}$ & $\begin{array}{l}-4.59 \\
10^{-3} \mathrm{mdyn}\end{array}$ & $\begin{array}{c}0.57^{6} \\
10^{-3} \AA\end{array}$ \\
\hline
\end{tabular}

${ }^{\mathrm{a}} \mathrm{By} \tilde{S}_{8}, \tilde{S}_{11}$.

'Neglects small $E_{u}, E_{z}$ contributions.

'Entirely due to $E_{k}$ vibrations. The appreciable torsional foreshortening component $F_{3}^{F}$ is cancelled identically by the other $\xi_{i}^{F}$ in the computation of $\left\langle S_{3}\right\rangle_{F}$.

dences are in terms quadratic in $\mathrm{CCH}$ bends.

Isotopic dependences of the vibrational stress components and mean displacements of rectilinear coordinates are listed in Table II for $\mathrm{C}_{2} \mathrm{H}_{8}$ and $\mathrm{C}_{2} \mathrm{D}_{6}$. Although the mass variations of the cubic constants $f_{i j k}$ do contribute to the isotope effects, the mass differences in the mean square amplitudes $\left\langle S_{i} S_{f}\right\rangle$ are rather more important. Representative values of $\left\langle S_{i} S_{j}\right\rangle$ are tabulated in Ref. 5 .

A decomposition of vibrational stress components is presented in Table III for the $\mathrm{C}-\mathrm{C}$ stretch, along with vibrational components of the foreshortening correction $\left(r_{z}-r_{z}\right)$. The vibrational effects on this internal coordinate are overwhelmingly localized in $\mathrm{CCH}$ bend contributions, which are, of course, strongly mass dependent. (The appreciable $\tilde{F}_{3}$ component from the $\mathrm{C}-\mathrm{C}$ stretching motion itself is not sensitive to hydrogen mass, however.) The effect of hydrogen mass on the $\mathrm{C}-\mathrm{C}$ bond stretch is a secondary isotope effect, the origin of which can plausibly be ascribed to geminal $\mathrm{H} \cdot \cdots \mathrm{C}$ nonbonded repulsions. These are enhanced by $\mathrm{CCH}$ vibrations as outlined elsewhere. ${ }^{5,11}$

\section{CONCLUSION}

In summary, we have separated cubic constants and vibrational stress and strain components into three classes with different physical meanings. None can be neglected in quantitative work. These can be further decomposed into interpretable components for diagnosing vibrational effects, if desired. In the computation of vibrational averages in molecular structure the present treatment permits nothing to be done that could not be done in the earlier treatments of Kuchitsu ${ }^{2,3}$ or Mills. ${ }^{4}$ The intermediate quantities encountered, how ever, have a more readily visualizable meaning, and natural curvilinear coordinates are handled more directly than previously.

\section{ACKNOWLEDGMENTS}

This work was supported by a grant from National Science Foundation. We are grateful to Professors Ian Mills and Kenneth Hedberg for performing calculations of the $\mathrm{L}$ tensor elements for $\mathrm{C}_{2} \mathrm{H}_{6}$ and for communicating valuable criticisms of the manuscript. We thank Mr. Scott Woehler for carrying out the $a b$ initio calculations of $\tilde{f}_{112}$ and $\tilde{f}_{221}$.

${ }^{1}$ Y. Morino, J. Nakamura, and P. W. Moore, J. Chem. Phys. 36, 1050 (1962).

${ }^{2}$ K. Kuchitsu, Bull. Chem. Soc. Jpn. 40, 505 (1967).

${ }^{3}$ For reviews see K. Kuchitsu and S. J. Cyvin, Molecular Structure and Vibrations (Elsevier, Amsterdam, 1972), p. 183; A. G. Robiette, Molecular Structure by Diffraction Methods (The Chemical Society, London, 1973), Vol. 1, p.160.

${ }^{4}$ A. R. Hoy, I. M. Mills, and G. Strey, Mol. Phys. 24, 1265 (1972).

${ }^{5}$ L. S. Bartell, S. Fitzwater, and W. J. Hehre, J. Chem. Phys. 63, 3042 (1975).

${ }^{6}$ P. Ehrenfest, Z. Phys. (Leipzig) 45, 455 (1927).

${ }^{7}$ L. S. Bartell, J. Chem. Phys. 38, 1827 (1963).

${ }^{8}$ I. M. Mills, J. Phys. Chem. 80, 1187 (1976).

${ }^{9}$ L. S. Bartell, S. Fitzwater, and W. J. Hehre, J. Chem. Phys. 68, 4750 (1975). Note that the geminal steric model entries in Table VI of this paper are in error for constants $F_{882}$ and $F_{11,11,2}$. These constants should be -0.082 rather than -0.218. We thank Professor Bernard Kirtman for pointing out our error.

${ }^{10}$ J. L. Ducan, Spectrochim. Acta 20, 1197 (1964).

${ }^{11}$ L. S. Bartell, Iowa State J. Sci. 36, 137 (1961). 